\title{
NO IMMEDIATE EFFECTS OF HIGHLY CUSHIONED SHOES ON BASIC RUNNING BIOMECHANICS
}

\author{
Naoko Aminaka, Kevin Arthur, John P. Porcari, Carl Foster, \\ Maria Cress, and Chelsea Hahn \\ University of Wisconsin - La Crosse, La Crosse, WI, USA
}

Original scientific paper

https://doi.org/10.26582/k.50.1.10

UDC: $796.422: 577$

\begin{abstract}
:
The aim of the study was to investigate the effects of highly cushioned shoes on running biomechanics. Sixteen recreational runners ( 8 males, 8 females) participated and ran at a self-selected pace across the force platform in the research laboratory wearing either the standard or highly cushioned shoes, in randomized order. Impact peak (IP), loading rate to IP (LR), active peak (AP), contact time (CT), strike index (SI), running velocity, and knee and ankle kinematics at initial contact (IC) and AP were recorded during the running trials. Overall, there was no effect of footwear on IP, LR, AP, CT and velocity ( $>>.05)$ with small effect sizes $(\mathrm{ES}<0.2)$. The highly cushioned shoes resulted in a more anterior foot strike pattern, based on the slightly higher $\mathrm{SI}(\mathrm{p}=.03, \mathrm{ES}=0.5)$, although the runners demonstrated a rearfoot strike pattern regardless of shoe condition. No kinematic differences were observed at IC or AP, across shoe conditions ( $\mathrm{p}>.05)$. Our results indicate that the highly cushioned shoes did not show immediate changes in running biomechanics.
\end{abstract}

Key words: kinematics, kinetics, running, footwear, extra cushion

\section{Introduction}

Running has increased in popularity in the last several decades. The total number of runners participating in running races in USA increased by $300 \%$ from 1990 to 2013 (Running USA, 2016). While the number of runners has declined from the peak in 2013, there are still approximately 17 million people who completed running races in the United States in 2015 (Running USA, 2016). With such a large number of people running, the risk and incidence of running-related injuries will likely continue. During a 12-month period of running, it has been reported that up to $90 \%$ of runners will suffer a running-related injury (Fields, Sykes, Walker, \& Jackson, 2010; Satterthwaite, Larmer, Gardiner, \& Norton, 1996; Van Gent, et al., 2007).

There are various intrinsic and extrinsic factors that may contribute to the risks of developing running-related injuries, such as history of previous injuries, training errors, improper biomechanics, environmental factors, and footwear (Fredericson \& Misra, 2007; Lopes, Hespanhol Junior, Yeung, \& Costa, 2012; Van Gent, et al., 2007; Wen, 2007). The amount, frequency, and timing of impact forces are considered as the major contributing factors in injury (Chambon, Delattre, Gueguen, Berton, \& Rao, 2014; Clarke, Frederick,
\& Cooper, 1983). While a variety of changes to footwear design have been made in attempts to reduce impact forces, midsole thickness has been studied extensively. Many studies have investigated the effects of varying running shoe midsole thickness, ranging from minimalist shoes/barefoot conditions to standard athletic shoes during running (Chambon, et al., 2014; Hamill, Russell, Gruber, \& Miller, 2011; Lafortune \& Hennig, 1992; Lieberman, et al., 2010; Perry, Ulbrecht, Derr, \& Cavanaugh, 1995; TenBroek, Rodrigues, Frederick, \& Hamill, 2014). These studies commonly agreed that there were lower impact forces, reduced loading rate, and decreased ankle dorsiflexion when comparing shod (any type of footwear) to a barefoot running condition. However, results appear inconclusive regarding how different amounts of midsole thickness may alter running biomechanics while shod. The effects of a highly cushioned shoe with a thick midsole is largely unknown.

A recent running shoe design has incorporated rocker soles (Boyer \& Andriacchi, 2009; Hutchins, Bowker, Geary, \& Richards, 2009; Kastenbauer, Sokol, Auinger, \& Irsigler, 1998; Kimel-Scott, Gulledge, Bolena, \& Albright, 2014; B. Nigg, Hintzen, \& Ferber, 2006; Perry, et al., 1995) along with the increased amount of midsole cushioning beyond that of standard shoes. These highly cush- 
ioned rocker shoes are marketed for distance and ultra-distance runners, and claim to be effective in reducing impact forces and loading rate due to an increased time of ground contact. These highly cushioned shoes also claim to promote more midfoot to forefoot strike patterns, which may also contribute to reduced impact forces. A recently published study has investigated the effects of the "maximalist" (highly cushioned) footwear on running biomechanics (Sinclair, Fau-Goodwin, Richards, \& Shore, 2016). The results from the study indicate no detrimental effects of the highly cushioned shoes when compared to the minimalist shoes. However, the benefits of the highly cushioned shoes still seem to be unclear, as no statistically significant findings were observed between the highly cushioned and conventional (traditional) cushioned shoes. Thus, it was felt that more research is necessary for further understanding of the potential benefits of highly cushioned shoes on running biomechanics in comparison with standard cushioned shoes.

Our purpose was to compare running kinetics and kinematics in highly cushioned versus standard running shoe conditions in healthy runners. Specifically, we were interested in how highly cushioned shoes affect impact forces, rate of loading, stance phase time, foot striking pattern, and knee and ankle joint kinematics during the stance phase of running. We hypothesized that the highly cushioned shoes would reduce forces (impact peak [IP] and active peak $[\mathrm{AP}])$, reduce loading rate (LR), and increase stance phase time (contact time [CT]). Also, we hypothesized that the highly cushioned shoes would achieve non-rearfoot strike patterns (higher strike index [SI]) and decrease ankle dorsiflexion angle at initial contact (IC), but would have no effect on knee flexion angles during stance.

\section{Methods}

\section{Participants}

Participants in this study were 16 volunteers (eight males, eight females) from the local commu-

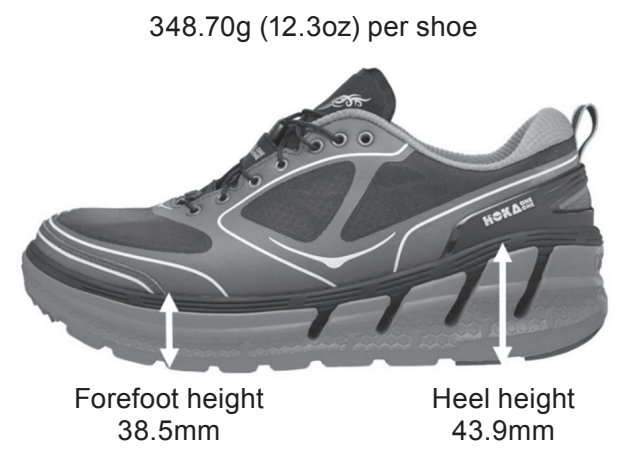

A. Highly cushioned shoes (HOKA ONE ONE $®$ Conquest) nity who ran at least six miles per week, and had not had a lower extremity orthopedic injury within three months prior to testing. Participants' demographics are shown in Table 1. Participants selfreported as being habitually rearfoot strikers and shod runners; however, the participants had never worn such highly cushioned shoes prior to the study. Participants provided written informed consent prior to undergoing any testing procedures. The University Institutional Review Board for the Protection of Human Subjects approved this study.

\section{Procedure}

During one data collection session, each participant ran under two shoe conditions (highly cushioned and standard running shoes), in random order. HOKA ONE ONE ${ }^{\circledR}$ Conquest (HOKA ONE ONE ${ }^{\circledR}$, Goleta, CA) shoes were used as the highly cushioned shoes (Figure 1A). These shoes had a thick midsole (heel height $43.9 \mathrm{~mm}$ in male, $41.7 \mathrm{~mm}$ in female; forefoot height $38.5 \mathrm{~mm}$ in male, $35.6 \mathrm{~mm}$ in female). The highly cushioned shoes weighed $348.70 \mathrm{~g}(12.3 \mathrm{oz})$ per shoe for males, and $297.67 \mathrm{~g}$ (10.5oz) per shoe for females. New Balance 481 shoes (New Balance, Boston, MA) were used as the standard running shoes (Figure 1B). The standard shoes weighed $267.0 \mathrm{~g}(9.42 \mathrm{oz})$ per shoe for males, and $195.2 \mathrm{~g}(6.89 \mathrm{oz})$ per shoe for females, and had a heel height of $32.0 \mathrm{~mm}$ for males and females, and the forefoot height of $16.0 \mathrm{~mm}$ for males and $15.0 \mathrm{~mm}$ for females. Prior to data collection, partic-

Table 1. Participant demographics $(N=16)$

\begin{tabular}{lc}
\hline Variables & M $\pm S D$ \\
\hline Age (year) & $27.9 \pm 13.13$ \\
Body height $(\mathrm{cm})$ & $174.7 \pm 6.51$ \\
Body mass $(\mathrm{kg})$ & $71.23 \pm 10.14$ \\
Weekly running distance (miles) & $28.6 \pm 21.3$ \\
Weekly running frequency (times) & $4.8 \pm 1.47$ \\
Average running speed (miles/hour) & $7.7 \pm 1.46$ \\
\hline
\end{tabular}

$\mathrm{M}=$ mean; $\mathrm{SD}=$ standard deviation.

$195.2 \mathrm{~g}(6.89 \mathrm{oz})$ per shoe

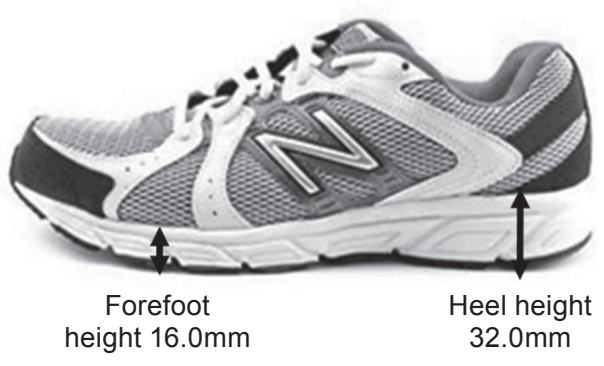

B. Standard shoes (New Balance 481)

Figure 1. Footwear types. Male models are referenced for each footwear type. 
ipants jogged/ran for five minutes at a self-selected pace on an indoor track wearing their own shoes. The participants then put on the first pair of shoes (either the highly cushioned or standard shoes) for the first testing condition. Retroflective markers were placed at the $1^{\text {st }}$ metatarsal head, $5^{\text {th }}$ metatarsal head, calcaneal tuberosity, lateral malleolus, medial malleolus, mid-tibia (shank), lateral knee joint line, medial knee joint line, mid-femur (thigh), greater trochanter of the femur, anterior superior iliac spine, posterior superior iliac spine, and sacrum, to capture joint kinematics. Following the marker setup as well as static and dynamic calibration, the medial markers at the knees and ankles were removed. The participant completed 5-10 practice trials running across the laboratory space (approximately 20 yards), to become familiarized with the shoes. After an adequate rest, the participants ran at a self-selected speed until five successful running trials were obtained. A trial was considered successful if an entire foot made contact with one of the force plates (2 AMTI OR6-7 Force platforms, AMTI, Watertown, MA). Running velocity was monitored using the horizontal velocity from the sacral marker at the time of initial foot contact with the force plate. Trials were discarded if 1 ) the entire foot did not make contact with one of the two force plates, 2) running velocity exceeded $\pm 5 \%$ of the participant's preferred running speed, and 3 ) the participant altered his or her gait in order to make full contact with the force plate. Participants were given a minimum of 30 seconds of rest between running trials. Once data collection with the first shoe condition was completed, a 15-minute rest period was given. Following the rest period, participants switched shoe conditions and repeated the testing procedure. Three dimensional kinematic data (Vicon, Centennial, CO) were recorded with 8 Bonita B3 and 2 Bonita B10 cameras at $200 \mathrm{~Hz}$ in Nexus 2.0. The kinetic data from the force plates were collected at $1000 \mathrm{~Hz}$. All data from the cameras and force plates were collected and processed through The MotionMonitor ${ }^{\mathrm{TM}}$ software (Innovative Sports Training, Chicago, IL). All kinematic and kinetic data were smoothed with a low pass, zero-lag, fourth-order Butterworth filter with a cut-off frequency of $20 \mathrm{~Hz}$.

All data were collected from the participant's dominant leg (right leg for all participants). The kinetic variables included IP, LR, and AP during the run. The IC with the force plate was determined when the vertical ground reaction force exceeded 20 Newtons $(\mathrm{N})$. The kinetic variables were normalized to each participant's body weight (BW) in $\mathrm{N}$. For the IP, the first peak of the vertical ground reaction force during stance was used. The LR was calculated using the $20-80 \%$ window between the IC and IP, and was reported in BW/s. The AP was the overall peak of the vertical ground reaction force during stance. The kinematic variables included CT, SI, and knee flexion/extension and ankle dorsiflexion/plantarflexion at IC and at AP. Positive values indicated knee flexion and ankle dorsiflexion, while negative values indicated knee extension and ankle plantarflexion. The SI was the measure of foot strike pattern, and was calculated using the methods introduced by Cavanaugh and Lafortune (1980). The SI $<33 \%$ indicated rear foot strike pattern, whereas the SI $>66 \%$ indicated nonrearfoot strike pattern (midfoot or forefoot).

\section{Statistical analysis}

Kinetic and spatiotemporal dependent variables included IP, LR, AP, CT, SI, and running velocity. Kinematics dependent variables included knee flexion angle at IC and AP, and ankle dorsi/ plantarflexion angle at IC and AP. For each group of variables (kinetic and kinematic), a separate repeated measures analysis of variance (ANOVA) was performed to identify any significant differences between shoe conditions (highly cushioned and standard shoes) and gender. The level of significance was set apriori at $\mathrm{p}<.05$. Cohen's d was calculated to indicate effect sizes (ES). An ES of 0.2 was determined as small, 0.5 as medium, and 0.8 as large effects (Portney \& Watkins, 2000). IBM SPSS Statistics version 22.0 (IBM Corporation, Armonk, NY) was used for all statistical analyses.

\section{Results}

\section{Kinetics and spatiotemporal variables}

The initial repeated measures ANOVA between shoe conditions and gender yielded no effects of gender on running biomechanics across the shoe conditions ( $p>.05)$. Therefore, data were collapsed across gender, and another repeated measures ANOVA was performed using a shoe condition as the independent variable.

There was no significant difference in the average running velocity between the two shoe conditions, with a small effect size $\left(\mathrm{HOKA}^{\circledR}=3.06 \pm 0.43 \mathrm{~m} / \mathrm{s} ;\right.$ Standard $=3.12 \pm 0.46 \mathrm{~m} / \mathrm{s}$; $\left.\mathrm{F}_{1,15}=1.984, \mathrm{p}=.179, \mathrm{ES}=-0.19\right)$. Additionally, CT was not different between the shoe conditions $\left(\mathrm{F}_{1,15}=2.416, \mathrm{p}=.141, \mathrm{ES}=0.39\right)$. The results for the kinetics and spatiotemporal variables are presented in Table 2. There was no effect of the shoe condition for IP $\left(\mathrm{F}_{1,15}=0.683, \mathrm{p}=.422, \mathrm{ES}=0.07\right)$ or for AP $\left(\mathrm{F}_{1,15}=3.417, \mathrm{p}=.084, \mathrm{ES}=-0.19\right)$. There was no statistically significant difference in the LR between the shoe conditions $\left(\mathrm{F}_{1,15}=0.224, \mathrm{p}=.643\right.$, $\left.\mathrm{ES}=-0.06\right)$. There was a significant difference in the SI between the shoe conditions, with a medium effect size $\left(\mathrm{F}_{1,15}=3.417, \mathrm{p}=.03, \mathrm{ES}=0.50\right)$. Although participants seemed to demonstrate a rearfoot strike pattern under both shoe conditions $(<33 \% \mathrm{SI})$, running in HOKA $^{\circledR}$ shoes resulted in a slightly more anterior foot strike pattern than running in standard shoes. 
Table 2. Kinetic and spatiotemporal data $(M \pm S D)$

\begin{tabular}{lccccc}
\hline & HOKA $^{\circledR}$ shoes & Standard shoes & F-value & $p$ & \multicolumn{2}{c}{ Effect size } \\
\hline IP (BW) & $1.54 \pm 0.31$ & $1.52 \pm 0.29$ & 0.683 & .422 & 0.07 \\
LR (BW/s) & $42.79 \pm 10.64$ & $43.44 \pm 10.98$ & 0.224 & .643 & -0.06 \\
AP (BW) & $2.48 \pm 0.22$ & $2.52 \pm 0.19$ & 3.417 & .084 & -0.19 \\
SI (\% foot length) & $20.12 \pm 10.63$ & $15.00 \pm 9.64$ & 12.854 & $.03^{*}$ & 0.50 \\
CT (s) & $0.28 \pm 0.057$ & $0.26 \pm 0.036$ & 2.416 & .141 & 0.39 \\
Vel (m/s) & $3.06 \pm 0.43$ & $3.12 \pm 0.46$ & 1.984 & .179 & -0.13 \\
\hline
\end{tabular}

Note: $\mathrm{SI}=$ strike index; $\mathrm{CT}=$ contact time; $\mathrm{IP}=$ impact peak; $\mathrm{LR}=$ loading rate; $\mathrm{AP}=$ active peak; Vel=velocity.

$\mathrm{SI}$ is expressed as the percentage of the foot length. IP and AP are expressed in units of body weight, and the LR is expressed as the IP (units of body weight) divided by the seconds.

${ }^{*}$ Indicates significant difference between shoe conditions $\left(\mathrm{HOKA}^{\circledR}\right.$ and Standard $)(p<.05)$.

Table 3. Comparison of joint angles at initial contact $(M \pm S D)$

\begin{tabular}{lccccc}
\hline & HOKA $^{\oplus}$ shoes & Standard shoes & F-value & $p$ & Effect size \\
\hline Knee flexion $\left({ }^{\circ}\right)$ & $7.05 \pm 6.05$ & $7.41 \pm 5.80$ & .189 & .672 & -0.06 \\
Ankle dorsiflexion $\left(^{\circ}\right)$ & $9.33 \pm 5.30$ & $8.73 \pm 6.57$ & .689 & .546 & 0.10 \\
Ankle inversion $\left(^{\circ}\right)$ & $4.71 \pm 3.36$ & $5.68 \pm 5.77$ & .698 & .421 & -0.20 \\
\hline
\end{tabular}

Table 4. Comparison of joint angles at active peak $(M \pm S D)$

\begin{tabular}{|c|c|c|c|c|c|}
\hline & HOKA $^{\circledR}$ shoes & Standard shoes & F-value & $\mathrm{p}$ & Effect size \\
\hline Knee flexion $\left({ }^{\circ}\right)$ & $36.60 \pm 3.87$ & $36.54 \pm 4.01$ & .008 & .929 & 0.01 \\
\hline Ankle dorsiflexion $\left({ }^{\circ}\right)$ & $21.38 \pm 3.17$ & $21.97 \pm 4.99$ & .328 & .579 & -0.14 \\
\hline Ankle inversion $\left({ }^{\circ}\right)$ & $-6.26 \pm 1.56$ & $-7.21 \pm 4.98$ & .448 & .517 & 0.25 \\
\hline
\end{tabular}

\section{Kinematics}

Kinematic data are presented in Tables 3 and 4. The knee and ankle joint angles at IC were unaffected by the shoe condition ( $\mathrm{p}>$.05). Similarly, there were no significant differences in knee and ankle joint angles between the shoe conditions at the time of AP ( $p>05)$.

\section{Discussion and conclusions}

Our purpose was to compare kinetic and kinematic data when running in highly cushioned versus standard running shoes. Our specific hypotheses were that 1$)$ highly cushioned $\left(\mathrm{HOKA}^{\circledR}\right)$ shoes would result in a lower IP and AP, reduced LR, and more non-rearfoot strike pattern compared to running in standard shoes; and 2) highly cushioned shoes would result in less ankle dorsiflexion, but no difference would occur in ankle inversion and knee flexion angles compared to running in standard shoes. Only a small part of the first hypothesis was supported in our study. HOKA ${ }^{\circledR}$ shoes resulted in a slightly more anterior foot strike pattern, although both shoe conditions still yielded rearfoot strike patterns. The other hypotheses, related to kinematics and kinetics, were not supported in the current study.
HOKA $^{\circledR}$ shoes have a rocker sole design with accentuated curvature of the sole compared to the standard shoes. This design may have resulted in the approximately 5\% anterior shift in SI in our study. A previous study that investigated rocker sole design observed changes in sagittal plane ankle kinematics, ankle joint moments and power, suggesting the possible injury-reducing benefits (Boyer \& Andriacchi, 2009). Contrarily, we did not find any significant kinematic or kinetic differences between the shoe conditions. The amount of curvature in the HOKA ${ }^{\circledR}$ shoes may be less than the curvature in the shoes used in the previously mentioned study (Boyer \& Andriacchi, 2009). Based on our results, the slight shift in SI may not be largely beneficial in changing the kinematic and kinetic variables.

Our results indicating no differences in kinetic variables across shoe conditions were not expected, as we hypothesized that high cushioning in the midsole would reduce the overall exerted force. It was thought that the extra thickness of the shoes may create a sense of protection or safety, that may allow a runner to contact the ground with a relatively higher force without experiencing pain or discomfort (Dinato, et al., 2015). While our results on the LR were supported by a few studies (Chambon, et 
al., 2014; Sinclair, et al., 2016), other studies have shown lower LRs (Lafortune \& Hennig, 1992; Lieberman, et al., 2010; Logan, Hunter, Hopkins, Feland, \& Pacell, 2010) with thicker sole shoes. Some studies have also demonstrated reduced impact forces while wearing extra cushion shoes (Lafortune \& Hennig, 1992; TenBroek, et al., 2014), while other studies did not demonstrate differences in impact forces among different cushion thickness or sole geometry (Chambon, et al., 2014; Hamill, et al., 2011; Sacco, et al., 2012). The combined results from the kinetic variables may indicate that there may be individual variation when it comes to force attenuation. Since we only measured the immediate effects of running in different shoe types, continued research on longer-term adaptation with the highly cushioned shoes is warranted. The particular type of highly cushioned running shoes (HOKA ${ }^{\circledR}$ shoes) were designed for ultra-distance (longer than the full marathon distance of $42.195 \mathrm{~km}$ ) runners, and therefore it would also be interesting to measure the effects of the highly cushioned shoes during a prolonged run.

Our results found no differences in knee and ankle kinematics at IC and at AP between the shoe conditions. Our results contradict our hypothesis, which was based on two other studies (Chambon, et al., 2014; TenBroek, et al., 2014) that did observe changes in the various lower extremity kinematics at initial contact and midstance. However, these studies compared kinematics with different shoes that had thickness ranging from that of regular athletic shoes to minimalist shoes or a barefoot running condition. Our study compared regular thickness athletic shoes to the highly cushioned shoes, and our results indicated that the highly cushioned shoes did not drastically change running kinematics compared to the standard shoes. Our results were similar to the results by Sinclair et al. (2016). Perhaps the features of the highly cushioned shoes used in our study may not require the runners to change their gait patterns, and allowed them to continue running with their typical kinematics and kinetics. Whether the continued usage of the highly cushioned shoes over a longer adaptation period would have changed the lower extremity kinematics is unknown. Studies indicate that habituation into barefoot running or minimalist shoes running can change running biomechanics (Azevedo, Mezencio, Amadio, \& Serrao, 2016). Although no study on habituation has been done using highly cushioned shoes, it may be worthwhile to investigate the habituation effects of highly cushioned shoes on running biomechanics over a longer period of time.
One of the limitations in this study is the small sample size $(\mathrm{N}=16)$, thus allowing a large standard deviation on our outcome variables. The selfselected running speed, the range of weekly running mileage and age, and inclusion of both sexes could also be factors resulting in the large standard deviation. However, we wanted to include the runners that would represent the general running population, which includes a wide range of weekly running mileage, speed, age, and other running-related factors. All of our runners were self-reported rearfoot strikers and habitually ran in footwear, to eliminate the possible biomechanical differences due to different foot strike patterns or lack of footwear. Another limitation could be that, for each running trial, the participant only ran for approximately 20 yards in a closed laboratory space. While this may not represent the actual running conditions, our intention was to obtain the force data along with the kinematic data. Running speed was monitored for each running trial, so that it would stay consistent (within 5\% of the self-selected pace for each individual) across the running trials. Not standardizing the individual running speed could be a limitation. However, we did not want to alter the runners' natural running biomechanics by standardizing the running speed. Future studies could investigate the kinematic and kinetic differences of different shoe cushioning at various standardized running speeds. The cushion materials for both shoes were inevitably different due to the different shoe manufacturers, and this could be another limitation of the study. While we could not control this difference, stiffness and other mechanical properties of the cushioning material can affect running biomechanics (Baltich, Maurer, \& Nigg, 2015; Cheung \& Ng, 2008; Dinato, et al., 2015; Dixon, 2008; B.M. Nigg, Bahlsen, Luethi, \& Stokes, 1987; B.M. Nigg, Baltich, Maurer, \& Federolf, 2012).

Our results could be interpreted two ways, when considering running shoe recommendations. First, our results indicate that extra cushioning did not help reduce the impact forces applied to the body, nor change the gait kinematics. However, our results can also be interpreted that the highly cushioned shoes did not cause drastic adverse changes to running biomechanics. Therefore, if a consumer finds the highly cushioned shoes to be more comfortable, the highly cushioned shoes most likely will not be detrimental. Further studies are warranted to investigate longer-term benefits of the highly cushioned shoes on running biomechanics. 


\section{References}

Azevedo, A.P.d., Mezencio, B., Amadio, A.C., \& Serrao, J.C. (2016). 16 weeks of progressive barefoot running training changes impact force and muscle activation in habitual shod runners. PLoS ONE, 11(12), e0167234. doi: 10.1371/ journal.pone.0167234

Baltich, J., Maurer, C., \& Nigg, B.M. (2015). Increased vertical impact forces and altered running mechanics with softer midsole shoes. PLOS ONE, 10(4), e0125196. doi: 10.1371/journal.pone.0125196

Boyer, K.A., \& Andriacchi, T.P. (2009). Changes in running kinematics and kinetics in response to a rockered shoe intervention. Clinical Biomechanics, 24(10), 872-876. doi: 10.1016/j.clinbiomech.2009.08.003

Cavanaugh, P.R., \& Lafortune, M.A. (1980). Ground reaction forces in distance running. Journal of Biomechanics, 13, 397-406.

Chambon, N., Delattre, N., Gueguen, N., Berton, E., \& Rao, G. (2014). Is midsole thickness a key parameter for the running pattern? Gait \& Posture, 40(1), 58-63. doi: 10.1016/j.gaitpost.2014.02.005

Cheung, R.T.H., \& Ng, G.Y.F. (2008). Influence of different footwear on force of landing during running. Physical Therapy, 88(5), 620-628.

Clarke, T., Frederick, E., \& Cooper, L. (1983). Effects of shoe cushioning upon ground reaction forces in running. International Journal of Sports Medicine, 4, 247-251.

Dinato, R.C., Ribeiro, A.P., Butugan, M.K., Pereira, I.L.R., Onodera, A.N., \& Sacco, I.C.N. (2015). Biomechanical variables and perception of comfort in running shoes with different cushioning technologies. Journal of Science and Medicine in Sport, 18, 93-97.

Dixon, S.J. (2008). Use of pressure insoles to compare in-shoe loading for modern running shoes. Ergonomics, 51(10), $1503-1514$.

Fields, K.B., Sykes, J.C., Walker, K.M., \& Jackson, J.C. (2010). Prevention of running injuries. Current Sports Medicine Reports, 9(3), 176-182.

Fredericson, M., \& Misra, A.K. (2007). Epidemiology and aetiology of marathon running injuries. Sports Medicine, 37(4-5), 437-439.

Hamill, J., Russell, E.M., Gruber, A.H., \& Miller, R. (2011). Impact characteristics in shod and barefoot running. Footwear Science, 3(1), 33-40.

Hutchins, S., Bowker, P., Geary, N., \& Richards, J. (2009). The biomechanics and clinical efficacy of footwear adapted with rocker profiles - Evidence in the literature. The Foot, 19, 165-170.

Kastenbauer, T., Sokol, G., Auinger, M., \& Irsigler, K. (1998). Running shoes for relief of plantar pressure in diabetic patients. Diabetic Medicine, 15, 518-522.

Kimel-Scott, D.R., Gulledge, E.N., Bolena, R.E., \& Albright, B.C. (2014). Kinematic analysis of postural reactions to a posterior translation in rocker bottom shoes in younger and older adults. Gait \& Posture, 39(1), 86-90. doi: 10.1016/j.gaitpost.2013.06.002

Lafortune, M.A., \& Hennig, E.M. (1992). Cushioning properties of footwear during walking: Accelerometer and force platform measurements. Clinical Biomechanics, 7, 181-184.

Lieberman, D.E., Venkadesan, M., Werbel, W.A., Daoud, A.I., D’Andrea, S., Davis, I.S., . . Pitsiladis, Y. (2010). Foot strike patterns and collision forces in habitually barefoot versus shod runners. Nature, 463, 531-535. doi: 10.1038 /nature 08723

Logan, S., Hunter, I., Hopkins, J.T., Feland, J.B., \& Pacell, A.C. (2010). Ground reaction force differences between running shoes, racing flats, and distance spikes in runners. Journal of Sports Science and Medicine, 9(1), 147-153.

Lopes, A.D., Hespanhol Junior, L.C., Yeung, S.S., \& Costa, L.O.P. (2012). What are the main running-related musculoskeletal injuries? Sports Medicine, 42(10), 891-905. doi: 10.1007/BF03262301

Nigg, B., Hintzen, S., \& Ferber, R. (2006). Effect of an unstable shoe construction on lower extremity gait characteristics. Clinical Biomechanics, 21, 82-88.

Nigg, B.M., Bahlsen, H.A., Luethi, S.M., \& Stokes, S. (1987). The influence of running velocity and midsole hardness on external impact forces in heel-toe running. Journal of Biomechanics, 20(10), 951-959. doi: 10.1016/00219290(87)90324-1

Nigg, B.M., Baltich, J., Maurer, C., \& Federolf, P. (2012). Shoe midsole hardness, sex and age effects on lower extremity kinematics during running. Journal of Biomechanics, 45(9), 1692-1697. doi: 10.1016/j.jbiomech.2012.03.027

Perry, J.E., Ulbrecht, J.S., Derr, J.A., \& Cavanaugh, P.R. (1995). The use of running shoes to reduce plantar pressures in patients who have diabetes. Journal of Bone and Joint Surgery, 77, 1819-1828.

Portney, L.G., \& Watkins, M.P. (2000). Foundations of clinical research: Application to practice (2 ${ }^{\text {nd }}$ ed.). Upper Saddle River, NJ: Prentice-Hall.

Running USA. (2016). 2016 State of the Sport - U.S. Road Race Trends. Retrieved March 1, 2017 from: http://www. runningusa.org/state-of-sport-us-trends-2015?returnTo=annual-reports

Sacco, I.C.N., Sartor, C.D., Cacciari, L.P., Onodera, A.N., Dinato, R.C., Pantaleao Jr., E., . . . Costa, P.H.C. (2012). Effect of a rocker non-heeled shoe on EMG and ground reaction forces during gait without previous training. Gait \& Posture, 36(2), 312-315. doi: 10.1016/j.gaitpost.2012.02.018 
Satterthwaite, P., Larmer, P., Gardiner, J., \& Norton, R. (1996). Incidence of injuries and other health problems in the Auckland Citibank marathon, 1993. British Journal of Sports Medicine, 30, 324-326.

Sinclair, J., Fau-Goodwin, J., Richards, J., \& Shore, H. (2016). The influence of minimalist and maximalist footwear on the kinetics and kinematics of running. Footwear Science, 8(1), 33-39. doi: 10.1080/19424280.2016.1142003

TenBroek, T.M., Rodrigues, P.A., Frederick, E.C., \& Hamill, J. (2014). Midsole thickness affects running patterns in habitual rearfoot strikers during a sustained run. Journal of Applied Biomechanics, 30, 521-528. doi: 10.1123/ jab.2012-0224

Van Gent, R.N., Siem, D., Van Middelkoop, M., Van Os, A.G., Bierma-Zeinstra, S.M.A., \& Koes, B.W. (2007). Incidence and determinants of lower extremity running injuries in long distance runners: A systematic review. British Journal of Sports Medicine, 41(8), 469-480.

Wen, D.Y. (2007). Risk factors for overuse injuries in runners. Current Sports Medicine Reports, 6, 307-313.

Submitted: April 6, 2017

Accepted: September 13, 2017

Published Online First: January 11, 2018

Correspondence to:

Naoko Aminaka, PhD, ATC

Assistant Professor, Exercise and Sport Science

1725 State Street, Mitchell Hall 135, La Crosse, WI

54601 USA

Phone: 608-785-8785

Fax: 608-785-8172

E-mail: naminaka@uwlax.edu 